\title{
Screening pre-renal transplant: Risk factors appear key but important questions remain
}

\author{
Jamieson M. Bourque, MD, MHS, ${ }^{a}$ Ami E. Iskandrian, $M D,{ }^{b}$ and Fadi G. Hage, \\ $M D^{\mathrm{b}, \mathrm{c}}$ \\ a Cardiovascular Division and the Cardiovascular Imaging Center, Department of Medicine, \\ University of Virginia Health System, Charlottesville, VA \\ b Division of Cardiovascular Disease, University of Alabama at Birmingham, Birmingham, AL \\ c Section of Cardiology, Birmingham Veterans Affairs Medical Center, Birmingham, AL
}

Received May 22, 2017; accepted May 24, 2017

doi:10.1007/s12350-017-0944-6

\section{See related article, pp. 2058-2068}

According to the United States Renal Data System, in 2014 there were 120,688 newly reported cases of endstage renal disease (ESRD) in the United States, and 678,383 patients were living with ESRD by the end of the year. ${ }^{1}$ During the same year, 17,914 kidney transplants were performed in the United States, the majority of which were from deceased donors (69\%). Thus, although kidney transplantation offers improved survival and quality of life compared to dialysis, the shortage of organs available for transplantation limits this therapy to a minority of patients that may benefit from it. Indeed, the kidney transplant waiting list continues to grow from year-to-year. In 2014, 88,231 dialysis patients were on the waiting list, a number several folds larger than annual kidney transplants. ${ }^{1}$ It is therefore not surprising that the median waiting time to deceased or living donor transplantation for first-time listing (waiting time is longer for subsequent listings) was 3.4 years in 2009 with some states having median waiting time in excess of 5 years. These realities pose ongoing challenges to renal transplant programs that need to factor in survival of the host in their allocation of organs.

Funding NIH 1K23HL119620.

Reprint requests: Jamieson M. Bourque, MD, MHS, Cardiovascular Division, Department of Medicine, University of Virginia Health System, 1215 Lee Street, Box 800158, Charlottesville, VA, 22908; jbourque@virginia.edu,jamieson2@gmail.com

J Nucl Cardiol 2018;25:2069-71.

$1071-3581 / \$ 34.00$

Copyright (C) 2017 American Society of Nuclear Cardiology.
Since cardiovascular disease is the number one cause of death in patients with ESRD as well as in those who have undergone kidney transplantation, cardiac evaluation is an essential part of the evaluation of ESRD patients prior to transplantation to reduce perioperative risk and later events that decrease the effective life of the transplanted organ. $^{2-4}$ Due to the conflicting recommendations regarding the cardiac evaluation of these patients by different guidelines, the American Heart Association and the American College of Cardiology Foundation (AHA/ACCF) published an expert consensus document on cardiac disease evaluation and management among kidney and liver transplantation candidates in 2012 that was endorsed by the American Society of Transplant Surgeons, American Society of Transplantation, and National Kidney Foundation. ${ }^{4}$ Since traditional Framingham risk factors do not perform well in this population, the multi-societal Statement identified eight risk factors for CAD specific to the transplantation population: age $>60$ years, diabetes, prior cardiovascular disease, hypertension, dyslipidemia, smoking, left ventricular hypertrophy, and $>1$ year on dialysis. The Statement endorsed noninvasive stress testing for the evaluation of kidney transplant candidates without active cardiac conditions regardless of functional status based on the presence of multiple risk factors identified above. The IIb evidence class and level of evidence $\mathrm{C}$ of this recommendation indicate that the utility of noninvasive stress testing in this population is less well established by evidence, and the recommendation is based on scientific opinion. Further, the Statement indicated that the number of risk factors that should be used to prompt testing is still to be determined but suggested that three or more risk factors appears to be reasonable.

In this issue of the Journal, Doukky et al report on a retrospective cohort study from Rush University 
Medical Center to validate the approach suggested by the AHA/ACCF Statement. ${ }^{5}$ In a cohort of 581 consecutive kidney transplant recipients, of whom 401 had pretransplant stress testing with myocardial perfusion imaging (MPI) and 90 had coronary angiography, the sum of AHA/ACCF risk factors was associated with obstructive coronary artery disease (CAD) by angiography and 30-day postoperative and long-term (mean follow-up $3.3 \pm 3.0$ years) cardiac death or non-fatal myocardial infarction. Among the 89 of 90 patients undergoing angiography who also had MPI, abnormal myocardial perfusion was associated with obstructive CAD independent of the sum of risk factors but not with 30-day post-operative cardiac events. For long-term outcomes, an abnormal MPI was independently associated with increased risk of cardiac events with a hazard ratio of 1.73, confidence interval 1.07-2.79. The authors noted an interaction between MPI findings and time to transplant with attenuation of the prognostic value of MPI the farther it was performed from transplantation. Given this, the authors analyzed their data in the subset of patients who underwent MPI within one year of transplantation. In these 224 transplant recipients, MPI provided incremental prognostic information in those with 3 or 4 AHA/ACCF risk factors (hazard ratio 3.17, confidence interval 1.001-10.02, Figures 2 and 3 in). ${ }^{5}$ Importantly, MPI was not associated with long-term outcomes in those with 0-2 or with 5-8 risk factors.

The authors should be congratulated on this valuable analysis that confirms the prognostic value of the AHA/ACCF risk factors in patients that underwent kidney transplantation and appears to validate the cutoff of three risk factors endorsed by the AHA/ACCF as a trigger for non-invasive stress testing with some caveats outlined below. Additionally, this study provides important preliminary data on the time-dependent attenuation in MPI predictive value, although further research is needed in this area.

The study has some limitations that should be appreciated to help interpret the findings. First, the study is retrospective in design and from a single institution which limits the generalizability of the findings. Second, the findings regarding obstructive CAD on angiography should be interpreted with caution due to classic referral or verification bias which tends to lower specificity of MPI. ${ }^{6}$ Third, the small number of subjects with two or fewer risk factors $(n=89)$ limits evaluation of this subgroup. Analysis is further exacerbated by the fact that not all patients had MPI and not all of those had MPI within 1 year of transplantation. Therefore, the finding that MPI is not prognostically useful in those with $<3$ risk factors is based on 41 subjects with normal MPI and 5 with abnormal MPI. Fourth, the analysis does not account for interventions that were introduced as a result of the stress tests, which may have attenuated the prognostic value of MPI. In addition, only the last MPI study prior to transplantation was considered in the analysis, which may have also impacted the findings. Fifth, the analysis is based on cardiac death rather than all-cause death. Although cardiac death may be best to evaluate cardiac risk, all-cause mortality is relevant in this situation to help avoid allocating valuable organs to hosts with short predicted survival irrespective of the cause of death. This problem was aggravated by the categorization of unknown deaths as non-cardiac rather than cardiac. Finally, only patients who eventually underwent transplantation were included in the analysis. No information is provided on candidates who underwent testing but did not receive transplantation, and this group may systematically differ as it would include those suffering cardiac events before receiving a transplant. ${ }^{7,8}$ Despite these limitations, this analysis provides important contributions to the knowledge on risk stratification prior to renal transplantation.

Doukky et al have increased our understanding in this challenging area, but many important questions remain. Is there value in incorporating $\mathrm{CT}$ coronary calcium scoring or CT coronary angiography in a hybrid approach? CT coronary angiography can identify obstructive disease with high sensitivity in the pretransplant population, but as with the general population is limited by poor positive predictive value, which may be particularly challenging in the ESRD population due to extensive coronary calcification. ${ }^{9,10}$ A hybrid SPECT/ CT angiography approach offers the advantages of both techniques but increases cost and radiation. Therefore, it will be key to identify in which patients this extensive testing is necessary. Likewise, CT coronary calcium scoring can help to identify the presence of CAD, although it may be best at guiding the intensity of medical therapy rather than determining need for angiography or gaging near-term and long-term risk in this population with known increased prevalence of vascular calcification. ${ }^{10-12}$ Moody et al recently showed that coronary artery calcium scoring does not add incremental prognostic information on top of MPI in ESRD patients being evaluated for renal transplantation. ${ }^{13}$

Additional improvements are likely possible within the use of MPI in this population. Advances in camera hardware and software protocols may improve diagnostic accuracy of SPECT MPI and extend it's utility. ${ }^{14}$ The high risk of this population may justify the increased cost of PET MPI, which has improved diagnostic accuracy over SPECT, although the costeffectiveness of such an approach remains to be demonstrated. ${ }^{15}$ In particular, PET MPI allows assessment of absolute myocardial blood flow and myocardial 
perfusion reserve (MPR). MPR has been shown to provide substantial additional risk stratification in multiple populations, including patients with CKD. ${ }^{16}$ Algorithms that include MPR may be able to improve the evaluation of patients for renal transplantation.

How should we incorporate these additional tools? The analysis by Doukky et al suggests that a varied approach based on the number of risk factors defined by the AHA/ACCF Statement may be a good potential strategy. ${ }^{4,5}$ Although Doukky et al suggest that those with 0-2 risk factors may not need MPI prior to transplant, this group may benefit from CT coronary calcium scoring or other testing to help guide management. Transplant candidates with 5-8 risk factors, who are at high risk independent of MPI results may benefit from hybrid SPECT/CT imaging or PET MPI which provides data on MPR. These approaches, although more costly, may provide valuable additional risk stratification in this subgroup and identify potential candidates for transplantation. Within those with 3-4 risk factors, there may also be subgroups that would benefit from further testing on top of MPI.

Additional remaining questions are the optimal time period for retesting and whether cardiovascular risk is reduced more with revascularization compared with medical therapy in the pre-renal transplant population. From a research standpoint, next steps that may be considered include: prospective analysis to assess the risk categories of 0-2, 3-4, and 5-8 risk factors in large cohorts; broadening the population studied to all those undergoing evaluation for renal transplant, not just those transplanted; randomized trials or prospective analyses of hybrid imaging strategies; longitudinal studies to identify optimal retesting periods; and prospective evaluation of outcomes based on imaging-directed medical therapy or coronary revascularization in asymptomatic renal transplant candidates.

\section{Disclosures}

Drs. Bourque and Hage have received research support from Astellas Pharmaceuticals. Dr. Iskandrian has nothing to disclose.

\section{References}

1. United States Renal Data System. 2016 USRDS annual data report: Epidemiology of kidney disease in the United States. Bethesda, MD: National Institutes of Health, National Institute of Diabetes and Digestive and Kidney Diseases; 2016.
2. Hage FG, Venkataraman R, Zoghbi GJ, Perry GJ, DeMattos AM, Iskandrian AE. The scope of coronary heart disease in patients with chronic kidney disease. J Am Coll Cardiol 2009;53:2129-40.

3. Rizk DV, Riad S, Hage FG. Screening for coronary artery disease in kidney transplant candidates. J Nucl Cardiol 2015;22:297-300.

4. Lentine KL, Costa SP, Weir MR, Robb JF, Fleisher LA, Kasiske $\mathrm{BL}$, et al. Cardiac disease evaluation and management among kidney and liver transplantation candidates: a scientific statement from the American Heart Association and the American College of Cardiology Foundation: endorsed by the American Society of Transplant Surgeons, American Society of Transplantation, and National Kidney Foundation. Circulation 2012;126:617-63.

5. Doukky R, Fughhi I, Campagnoli T, Wassouf M, Kharouta M, Vij A, et al. Validation of a clinical pathway to assess asymptomatic renal transplant candidates using myocardial perfusion imaging. $\mathrm{J}$ Nucl Cardiol 2017. doi:10.1007/s12350-017-0901-4.

6. Rozanski A, Diamond GA, Berman D, Forrester JS, Morris D, Swan HJ. The declining specificity of exercise radionuclide ventriculography. N Engl J Med 1983;309:518-22.

7. Hage FG, Smalheiser S, Zoghbi GJ, Perry GJ, Deierhoi M, Warnock D, et al. Predictors of survival in patients with end-stage renal disease evaluated for kidney transplantation. Am J Cardiol 2007; 100:1020-5

8. Doukky R, Fughhi I, Campagnoli T, Wassouf M, Ali A. The prognostic value of regadenoson SPECT myocardial perfusion imaging in patients with end-stage renal disease. J Nucl Cardiol 2017;24:112-8.

9. Di Carli MF, Dorbala S, Curillova Z, Kwong RJ, Goldhaber SZ, Rybicki FJ, et al. Relationship between CT coronary angiography and stress perfusion imaging in patients with suspected ischemic heart disease assessed by integrated PET-CT imaging. J Nucl Cardiol 2007; 14:799-809.

10. Winther S, Svensson M, Jorgensen HS, Bouchelouche K, Gormsen LC, Pedersen BB, et al. Diagnostic performance of coronary CT angiography and myocardial perfusion imaging in kidney transplantation candidates. JACC Cardiovasc Imaging 2015;8:553-62.

11. McClelland RL, Jorgensen NW, Budoff M, Blaha MJ, Post WS, Kronmal RA, et al. 10-Year coronary heart disease risk prediction using coronary artery calcium and traditional risk factors: Derivation in the MESA (multi-ethnic study of atherosclerosis) with validation in the HNR (Heinz Nixdorf Recall) study and the DHS (Dallas heart study). J Am Coll Cardiol 2015;66:1643-53.

12. Hage FG, Iskandrian AE. Multimodality imaging for CAD detection before renal transplantation. JACC Cardiovasc Imaging 2015;8:563-5.

13. Moody WE, Lin EL, Stoodley M, McNulty D, Thomson LE, Berman DS, et al. Prognostic utility of calcium scoring as an adjunct to stress myocardial perfusion scintigraphy in end-stage renal disease. Am J Cardiol 2016;117:1387-96.

14. Slomka PJ, Dey D, Duvall WL, Henzlova MJ, Berman DS, Germano G. Advances in nuclear cardiac instrumentation with a view towards reduced radiation exposure. Curr Cardiol Rep 2012;14:208-16.

15. Bateman TM, Heller GV, McGhie AI, Friedman JD, Case JA, Bryngelson JR, et al. Diagnostic accuracy of rest/stress ECG-gated Rb-82 myocardial perfusion PET: Comparison with ECG-gated Tc-99m sestamibi SPECT. J Nucl Cardiol 2006;13:24-33.

16. Murthy VL, Naya M, Foster CR, Hainer J, Gaber M, Dorbala S, et al. Coronary vascular dysfunction and prognosis in patients with chronic kidney disease. JACC Cardiovasc Imaging 2012;5:1025-34. 